\title{
Numerical analysis of sprays with an advanced collision model
}

\author{
M. Sommerfeld ${ }^{* 1,2}$ and S. Lain ${ }^{2}$ \\ ${ }^{1}$ Institut Verfahrenstechnik, Otto-von-Guericke-University Magdeburg, \\ Zeppelinstraße 1, D-06130 Halle (Saale), Germany \\ ${ }^{2}$ Department Mechanical Engineering, Universidad Autónoma de Occidente, Cali, Colombia \\ *Corresponding author: martin.sommerfeld@ovgu.de
}

\begin{abstract}
Modelling of collisions between liquid droplets in the frame of a Lagrangian spray simulation has still many open issues, especially when considering higher viscous droplets and if colliding droplets have a large size difference. A generalisation of the collision maps is attempted based on the behaviour of characteristic points, namely the triple point where bouncing, coalescence and stretching separation coincide and the critical Weber-number where reflexive separation first occurs in head-on collisions. This is done by correlating experimental data with respect to the Capillary number with the Ohnesorge-number for the triple point and the critical Weber-number is also well described by a correlation the Ohnesorge-number. Based on these results the boundary line between stretching separation and coalescence is found by adapting the Jiang et al. (1992) correlation. For the upper boundary of reflexive separation the shifted Ashgriz and Poo (1990) correlation is used. It was however so far not possible to predict the lower bouncing boundary through the Estrade et al. (1999) boundary line correctly. The proposed boundary-line models were validated for various liquid, however still considering only a size ratio of one. With the developed three-line boundary model Euler/Lagrange numerical calculations for a simple spray system were conducted and the droplet collisions were analysed with respect to their occurrence. Droplet collision modelling is performed on the basis of the stochastic droplet collision model, also considering the influence of impact efficiency, which so far was neglected for most spray simulations. The comparison with measurements showed reasonable good agreement for all properties.
\end{abstract}

\section{Keywords}

Droplet collisions, stochastic collision model, impact efficiency, collision outcomes, Euler/Lagrange calculations, spray simulations.

\section{Introduction}

In numerous technical and industrial spraying systems higher viscous liquids, suspensions or solutions are being atomised. Examples are liquid fuels (including bio-fluids) in combustion systems, liquid melts or other mineral melts in the field of materiel science and spray drying of foodstuff or pharmaceutical materials. Therefore, in a numerical calculation of such spraying systems, mostly done with an Euler/Lagrange approach, the resulting different liquid properties have to be accounted for. Here the focus is related to droplet collision modelling. Hence, established droplet collision models have to be generalized regarding liquid properties, mainly viscosity and surface tension. Since colliding droplets may have considerable different size also the droplet size ratio is an important parameter to be considered in droplet collision modelling.

In order to model droplet collisions in the frame of the Lagrangian droplet parcel concept, where only pointmasses are tracked, applied to spraying systems several elementary processes have to be considered (Sommerfeld and Kuschel 2016). The first step is the detection of possible collisions between two droplets. In order to insure that during a Lagrangian tracking time step only binary collisions can occur, the Lagrangian time step needs to be smaller than the reciprocal of the collision frequency (see e.g. Sommerfeld et al. 2008, Crowe et al. 2012). The collision detection may be done in three ways (Sommerfeld 2017), a fully deterministic and geometrical reconstruction of collision trajectories (e.g. Sundaram and Collins 1996), a deterministic-stochastic parcel collision model which requires to identify the probability of collision between any pair of parcels within an computational cell (O'Rourke 1981) and the fully stochastic droplet collision model (Sommerfeld 2001). This stochastic model does not require any knowledge about neighbouring droplets, but creates a fictitious collision partner out of the local droplet population. At this level these collision detection approaches assume $100 \%$ impact efficiency. However in a spray with a wide droplet size distribution, which also may be modified by coalescence, the smaller droplets might move around the larger ones with the relative velocity. This impact efficiency is the area from where the droplets are coming and hit the larger droplet, called collector, divided by the collector crosssection. Thereby the effective collision frequency is drastically reduced. When only considering inertial impact, the 
impact efficiency may be easily obtained through correlations depending on the relative Stokes number and the collector droplet Reynolds number (Ho and Sommerfeld 2002, Sommerfeld and Lain 2009). Resulting from that, the impact point of the small on the surface of the large droplet has to be determined giving the impact parameter B. With the known instantaneous relative velocity between colliding droplets also the collision Weber-number is determined. The next step is the identification of the collision scenario and the outcome on the basis of collision maps (Figure 1). The decision about which type of collision is occurring (i.e. bouncing, coalescence or stretching and reflexive separation), is based on analytical or theoretical derived boundary lines (see Figure 1). The properties of the droplets formed depend on the collision type at hand and have to be calculated based on mass and momentum balances (i.e. number of droplets, droplet sizes and their new velocities).

Very often spray computations are done by only considering one boundary line between coalescence and grazing collision, which involves bouncing and stretching separation (Figure 1). This approach was first suggested by O`Rourke (1981) using the boundary line derived theoretically by Brazier-Smith et al. (1972). The Euler/Lagrange approach was applied to spray calculations using this simplified collision map in various studies (e.g. Gavaises et al. 1996, Rüger et al. 2000, Guo et al. 2004, Nijdam et al. 2006). Composite collision maps applied to spray simulations, including also diesel sprays (Post and Abraham 2002, Mannannur and Reitz 2007), consider three boundary lines; namely the lower boundary of bouncing derived by Estrade et al. (1999), boundary between coalescence and stretching separation based on Brazier-Smith et al. (1972) and the boundary between reflexive separation and coalescence suggested by Ashgriz and Poo (1990). However these boundary lines cannot reflect the regimes observed for Diesel droplet collisions (Chen et al. 2016). This again shows the need for more general boundary lines which may be applied to different liquids.

In our previous studies an attempt was made to develop models which describe the collision outcome based on collision maps with boundary lines accounting for viscosity variations (Sommerfeld 2016). These new correlations could be developed by thorough experiments (Kuschel and Sommerfeld 2013, Sommerfeld and Kuschel 2016) and use the shift of the triple point (Coincidence of bouncing, coalescence and stretching separation) and the critical We-number (beginning of reflexive separation for head-on collisions) with variation of viscosity. The motion of the triple point showed a relative clear correlation with the Ohnesorge-number, which includes only droplet size and liquid properties. The boundary line coalescence-stretching separation was fitted by the Jiang et al. (1992) correlation, adapting the two involved model constants. These again could be well correlated with the Ohnesorgenumber for pure fluids and solutions separately (Sommerfeld 2016). In this contribution the data base for constructing the correlations for the triple point location and the critical We-number is extended based on recent studies and experiments (see also Sommerfeld 2017).

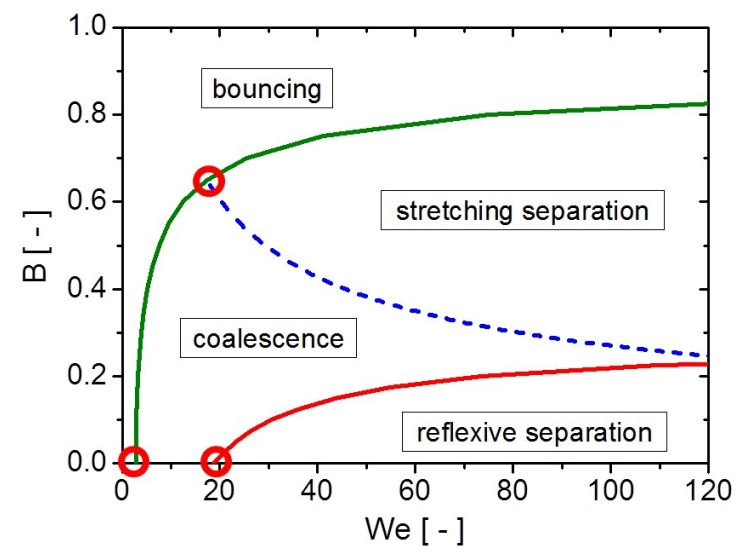

Figure 1: Typical droplet collision map where the non-dimensional impact parameter $B$ is plotted versus collision Weber-number We including boundary lines between collision outcomes and three characteristic points.

The resulting adaptive three-boundary line collision maps are first compared with collision maps obtained from experiments for different droplet liquids. Then the performance of the three boundary line collision maps is analysed for numerical calculation of turbulent sprays using the Euler/Lagrange approach. A comparison with the experiments of Rüger et al. (2000) is also shown for validation.

\section{Theoretical boundary lines}

Based on an energy balance Estrade et al. (1999) developed the lower boundary of bouncing. For that it was assumed that the initial droplet kinetic energy of the deformation region does not exceed the surface energy difference to produce a certain deformation. This derivation neglects any dissipation effects but includes the 
influence of droplet size ratio. Moreover, a shape factor is included in the correlation, depending on the degree of deformation during the bouncing process. Hence the shape factor should be depending on liquid dynamic viscosity. The correlation of Estrade et al. (1999) is given by:

$W e=\frac{\Delta\left(1+\Delta^{2}\right)(4 \Psi-12)}{\chi(\cos (\arcsin B))^{2}}$

$$
\begin{array}{llrl}
\chi & =1-0.25(2-\tau)^{2}(1+\tau) & & \text { if }: \tau>1 \\
\chi & =0.25 \tau^{2}(3-\tau) & & \text { if }: \tau \leq 1 \\
\tau & =(1-B)(1-\Delta) &
\end{array}
$$

The specified value of the shape factor was $\Psi=3.351$ fitting the data for ethanol at two size ratios. In previous studies it was aimed to adapt the bouncing boundary through the shape factor, which is somehow related to viscosity effects (Sommerfeld and Kuschel 2016). Such an adaptation was successful for higher Weber-numbers, but not below the triple point. The variation of the bouncing boundary in dependence of the size ratio is shown in Figure 2 a). With decreasing size ratio $\Delta=d_{S} / d_{L}$ the boundary line is slightly shifted upward and to the left in accordance with the measurements presented by Estrade et al. (1999). However, the question is whether the proposed correlation is also valid for small size ratios which are likely to be found in spraying systems as will be shown below.

Also the effect of the shape factor on the boundary line was analysed showing a rather strong dependence (Figure 2 b). A small value of $\Psi$ implies also a smaller deformation and hence lower shape factors. This is in accordance with measurements of Sommerfeld and Kuschel (2016) where with increasing viscosity, also associated with reduced deformation, the bouncing boundary is shifted upward.
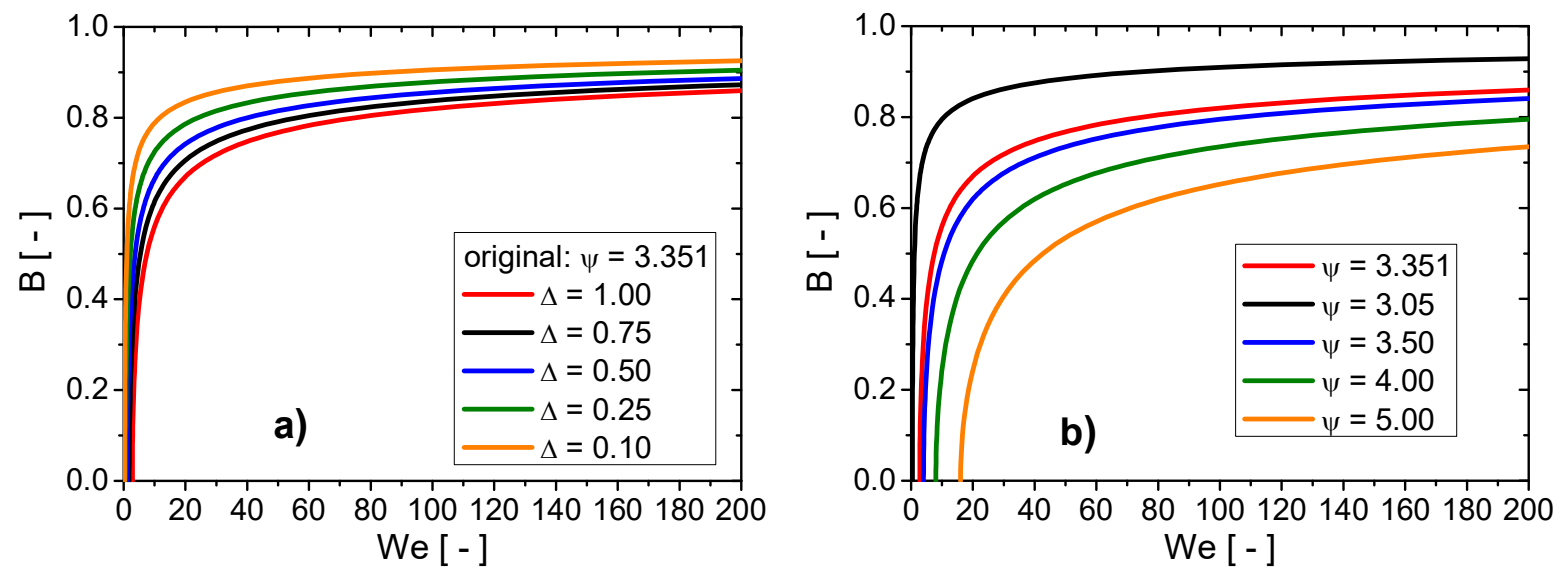

Figure 2: Collision map with Estrade et al. (1990) bouncing boundary lines showing the effect of (a) droplet size ratio $\Delta=d_{S} / d_{L}$ (shape factor $\psi=3.351$ ) and the influence of $(b)$ the shape factor.

The boundary line between coalescence and stretching separation was derived in a number of studies, namely Brazier-Smith et al. (1972), Ashgriz and Poo (1990) and Jiang et al. (1992). Brazier-Smith et al. (1972) assumed that if the rotational energy exceeds the surface energy required to reform two new droplets from the coalesced droplets, separation will occur. Also in this correlation the size ratio $\Delta$ was considered. The Brazier-Smith et al. (1972) correlation is very often used in spray calculations, just separating coalescence and grazing collisions (O'Rourke 1981), which is called here single line boundary model. The correlation of Brazier-Smith et al. (1972) for the boundary line is given by:

$W e=\frac{4.8}{B^{2}}\left(\left(\frac{1}{\Delta}\right)^{3}-2.4\left(\frac{1}{\Delta}\right)^{2}+2.7\left(\frac{1}{\Delta}\right)\right)$

The energy balance of Ashgriz and Poo (1990) assumes that stretching separation occurs if the total effective stretching energy is larger than the surface energy of the small interaction region. Also in this correlation the size ratio $\Delta$ was included and validated by experiments for water.

$W e=\frac{4\left(1-\Delta^{3}\right)^{2}\left\{3(1+\Delta)(1-B)\left(\Delta^{3} \phi_{S}+\phi_{L}\right)\right\}^{1 / 2}}{\Delta^{2}\left\{\left(1+\Delta^{3}\right)-\left(1-B^{2}\right)\left(\phi_{S}+\Delta^{3} \phi_{L}\right)\right\}}$ 


$$
\begin{aligned}
& \phi_{S}=\left\{\begin{array}{ll}
1-\frac{1}{4 \Delta^{3}}(2 \Delta-\tau)^{2}(\Delta+\tau) & \text { for } h>0.5 d_{S} \\
\frac{\tau^{2}}{4 \Delta^{3}}(3 \Delta-\tau) & \text { for } h<0.5 d_{S}
\end{array} \quad \phi_{L}= \begin{cases}1-\frac{1}{4}(2-\tau)^{2}(1+\tau) & \text { for } h>0.5 d_{L} \\
\frac{\tau^{2}}{4}(3-\tau) & \text { for } h<0.5 d_{L}\end{cases} \right. \\
& \mathrm{h}=\frac{1}{2}\left(\mathrm{~d}_{\mathrm{L}}+\mathrm{d}_{\mathrm{S}}\right)(1-\mathrm{B}) ; \quad \tau=(1-\mathrm{B})(1+\Delta)
\end{aligned}
$$

Finally the boundary line developed by Jiang et al. (1992) is based on a momentum balance and therefore includes dissipation effect. Hence, this is the only correlation which may be used to consider viscosity effects, as for example done by Gotaas et al. (2007) and Sommerfeld and Kuschel (2016).

$$
B=\frac{C_{a}}{W e^{1 / 2}}\left[1+C_{b} \frac{\mu}{\sigma}\left(\frac{\rho d_{1}}{\sigma}\right)^{1 / 2}\right]
$$

The analysis of Gotaas et al. (2007) suggested to use for the involved constants the values $C_{a}=2.33$ and $C_{b}=$ $0.41\left(\mathrm{~m}^{2} / \mathrm{s}^{2}\right)$. Since the correlation of Jiang et al. (1992) includes properties of the liquid (i.e. dynamic viscosity, surface tension and density) as well as the small droplet diameter it could be successfully used for liquids with increasing viscosity with the above set of model constants (Gotaas et al. 2007). In the work of Sommerfeld and Kuschel (2016) it could be shown that the model constants may be correlated reasonably well with the relaxation velocity $U_{\text {relax }}=\sigma / \mu$. The Jiang et al. (1992) correlation does not include the droplet size ratio, but the diameter of the small droplets. However, changing the droplet size over an order of magnitude in a relevant size range did not show a large variation of the boundary line.

The different coalescence/stretching boundary lines are compared in Figure 3. The correlations of Brazier-Smith et al. (1972) with size ratio one and Jiang et al. (1992) do not show any large difference. Larger are the deviations to the Ashgriz and Poo (1990) correlation for We smaller than 20, since it approaches the point We $=0$ and $\mathrm{B}=$ 1.0. Decreasing the size ratio in the Ashgriz and Poo (1990) correlation, results in a turning of the line around this point, yielding higher impact parameters $B$ for stretching separation to occur (Figure 3). This is not quite the trend observed in experiments where an upward and right shift of the boundary line is observed. On the other hand the Ashgriz and Poo (1990) correlation has never been validated for size ratios below $\Delta=0.5$. Here also the correlation shows some obscure behaviour for larger We.

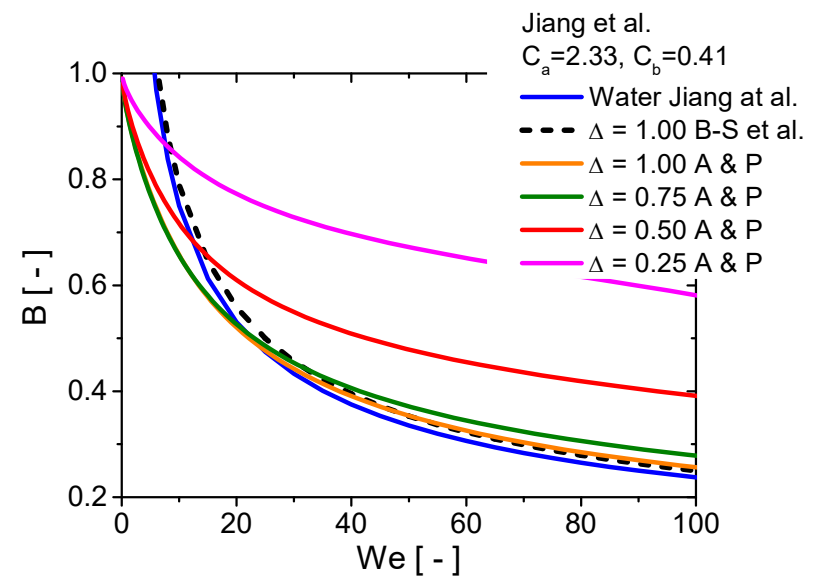

Figure 3: Different boundary lines for stretching coalescence transition in the collision map; Brazier-Smith et al. (1972) model with $\Delta=1.0$, Jiang et al. (1992) model for water with standard constants and Ashgriz and Poo (1990) model with decreasing droplet size ratio $\Delta$ (droplet size $750 \mu \mathrm{m}$ ).

Finally the boundary line between coalescence and reflexive separation is considered. In spray computations mostly the correlation also proposed by Ashgriz and Poo (1990) is used, given below:

$W e_{C}=3\left[7\left(1+\Delta^{3}\right)^{2 / 3}-4\left(1+\Delta^{2}\right)\right] \frac{\Delta\left(1+\Delta^{3}\right)^{2}}{\Delta^{6} \eta_{S}+\eta_{L}}$ 


$$
\begin{aligned}
& \eta_{\mathrm{S}}=2(1-\xi)^{2}\left(1-\xi^{2}\right)^{1 / 2}-1 \\
& \eta_{\mathrm{L}}=2(\Delta-\xi)^{2}\left(\Delta^{2}-\xi^{2}\right)^{1 / 2}-\Delta^{3} \\
& \xi=(1 / 2) \mathrm{B}(1+\Delta)
\end{aligned}
$$

This correlation also neglects viscosity but includes droplet size ratio. For $\Delta=1.0$ the critical Weber-number is about 18.7 as determined from many experimental studies for water. With decreasing size ratio the boundary line is shifted remarkably to the right, to higher We. However, this is not in accordance with the studies of Tang et al. (2012) where $\Delta=0.4$ results in a shift to $W_{e_{c}} \sim 40$. A shift of this boundary line to the right was also observed by Kuschel and Sommerfeld (2013) and Sommerfeld and Kuschel (2016) when increasing the dynamic viscosity of the liquid, similar to the work of Gotaas et al. (2007). This shift of the critical Weber number could be quite well correlated in dependence of the Ohnesorge number as well as the capillary number (Sommerfeld and Kuschel 2016).

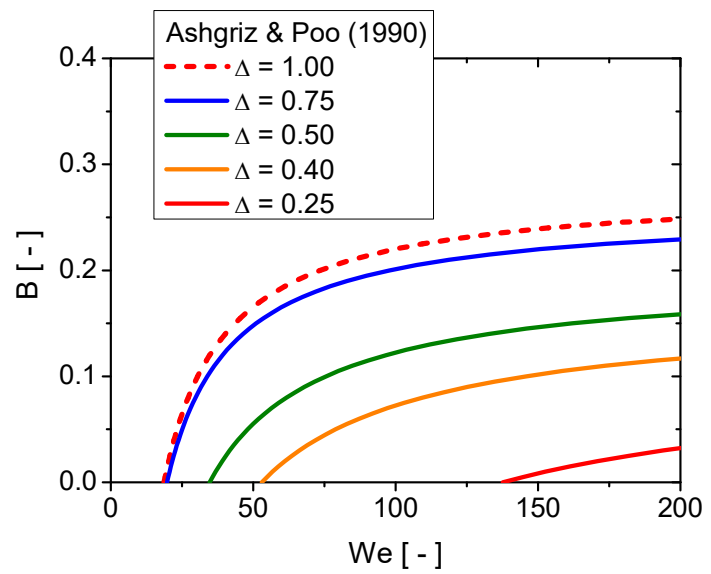

Figure 4: Boundary lines for coalescence/reflexive separation according to Ashgriz and Poo (1990) with decreasing size ratio $\Delta$.

\section{Euler/Lagrange approach}

The numerical scheme adopted to simulate the dispersed two-phase flow developing in a spraying system is the fully coupled stationary and three-dimensional Euler/Lagrange approach (Rüger et al. 2000, Lain and Sommerfeld 2008). The fluid flow was calculated based on the Euler approach by solving the Reynolds-averaged conservation equations on a fixed grid in connection with the standard $k-\varepsilon$ turbulence model equations. The effect of the dispersed phase on the fluid flow was accounted for by appropriate source terms in the momentum equations as well as the conservation equations for turbulent kinetic energy and dissipation rate, i.e., full two-way coupling.

The dispersed droplet phase is simulated by the Lagrangian approach, which implies that the spray is generated by tracking a huge number of droplet parcels through the beforehand calculated flow field. This requires the solution of the equations of the motion for each computational particle or parcel, being a representative of a number of real droplets with the same properties. These equations include the droplet inertia, drag and gravitybuoyancy. The Basset history term, the added mass and the fluid inertia are negligible for high ratios of droplet to gas density. The drag coefficient used in droplet tracking is that of Schiller and Naumann (1933).

For solving the partial differential equations of droplet motion a dynamic time step is used which is automatically adjusted according to the local (droplet location within the grid) governing time scales such as particle response time, integral time scale of turbulence and inter-particle collision time. The maximum tracking time step is selected to be $20 \%$ of the minimum of these time scales.

In order to account for turbulence effects on droplet motion, the instantaneous fluid velocity components along the particle trajectory are determined from the local mean fluid velocity interpolated from the neighbouring grid points and a fluctuating component generated by a single-step isotropic Langevin model described by Sommerfeld et al. (2008) and thoroughly validated in Sommerfeld (1996). In this model the fluctuation velocity is composed of a correlated part from the previous time step and a random component drawn from a Gaussian distribution function. The degree of correlation depends on the turbulent particle Stokes number $\mathrm{St}_{\mathrm{T}}$ and is calculated using appropriate time and length scales of turbulence estimated from the k- $\varepsilon$ turbulence model.

As mentioned above, two-way coupling considers the momentum transfer from the dispersed phase to the continuous phase and vice versa through appropriate source terms in the momentum equations and the conservation equations of turbulent kinetic energy and the dissipation rate. The source terms are accumulated for each control volume during the Lagrangian tracking procedure applying ensemble and temporal averaging 
(Sommerfeld 2017). The momentum sources are obtained from the change of particle velocity during its path through a control volume (Sommerfeld et al. 2008). An under-relaxation approach is used when introducing the source terms in the conservation equations of the fluid flow (for details see Kohnen et al. 1994, Lain and Sommerfeld 2012). Hence, a sequential calculation of fluid flow and particle phase is performed until the coupled hybrid system has converged. For the present calculations typically about 25 to 55 coupling iterations with an under-relaxation factor between 0.1 and 0.5 were necessary in order to yield convergence of the Euler-Lagrange coupling. This convergence behaviour was clearly demonstrated by Lain and Sommerfeld (2012).

\section{Stochastic droplet collision model}

For modelling droplet collisions in a spray the numerically efficient stochastic collision model (Sommerfeld 2001) combined with the impact efficiency (Ho and Sommerfeld 2002) is used. This model does not require searching for collision partners among surrounding droplets, rather during the tracking of the considered parcel a fictitious collision partner is drawn randomly from the local population in each time step (Sommerfeld 2001). This however requires that for each computational cell the droplet phase properties have to be made available, namely, concentration, droplet size distribution, and droplet size-velocity correlations with mean- and rms-values, droplet size-temperature correlations and so forth. From these distributions the properties of the fictitious droplet are drawn with size, velocity and temperature, by a stochastic process. The fictitious droplet velocity fluctuation needs however to be obtained through a pair-correlation with the real particle which depends on the particle Stokes number (i.e. the ratio of particle response time to the integral time scale of turbulence). This accounts for the effect that the velocities of responsive droplets are correlated through turbulence, whereas inertial particles have completely uncorrelated velocities (Sommerfeld 2001, Sommerfeld et al. 2008). Having all information about the collision partners the collision probability can be calculated:

$P=\frac{\pi}{6}\left(d_{S}+d_{L}\right)^{2}\left|\overrightarrow{u_{S}}-\overrightarrow{u_{L}}\right| n_{d} \Delta t_{L}$

If a random number is smaller than this probability a collision occurs. It should be mentioned again that the mean droplet collision time is an additional criterion for limiting the Lagrangian time step $\Delta \mathrm{t}_{\mathrm{L}}$ in order to insure only binary collisions, i.e. the collision probability is smaller than one. The collision itself is then calculated in a coordinate system where the fictitious particle is stationary. Thereby the collision point on the particle surfaces can be randomly determined (Sommerfeld 2001) and the lateral displacement $b$ of the collision partners is known. Such a stochastic model is numerically very efficient and the algorithm can be easily parallelised.

An essential element which has to be integrated in the collision detection is the impact efficiency which is relevant for a collision between small and large droplets (Ho and Sommerfeld 2002). Up to now the reduction of the collision rate in sprays through the impact efficiency has never been considered. In such a situation the smaller droplet might move around the larger one with the relative velocity field, which is a quite frequent phenomenon in sprays due to the wide size spectrum. Effectively, this results in a reduction of collision cross-section and hence collision rate, since often larger droplets will capture smaller droplets due to the existence of a high relative velocity (see Figure 5). This phenomenon may be described in a coordinate system where the larger collector droplet is stationary. Considering only inertial effects, the impact efficiency is then defined as the ratio of the circular cross-section from which the small droplets just will hit the larger droplet to the effective collector crosssection. Accounting also for the so-called blocking effect this cross-section diameter is the sum of the large and small droplet diameters (Figure 5). In the work of Schuch and Löffler (1978) the impact efficiency for inertial effects was calculated in dependence of the relative Stokes number which is the Stokesian relaxation time of the small droplet to the time it needs to pass the collector droplet with the relative velocity (Ho and Sommerfeld 2002):

$S t_{\text {rel }}=\frac{\tau_{P}}{T_{\text {Pass }}}=\frac{\rho_{S} d_{S}^{2} u_{r e l}}{18 \mu d_{L}}$

here the $d_{S}$ and $d_{L}$ refer to the diameters of the small and large droplet, respectively, $\rho$ is the small droplet density and $u_{\text {rel }}$ is the instantaneous relative velocity.

The impact efficiency, as defined below, is related to the relative Stokes number using the two parameters a and $b$, which depend on the collector droplet Reynolds number (Schuch and Löffler 1978):

$\eta=\left(\frac{2 Y_{C}}{d_{S}+d_{L}}\right)^{2}=\left(\frac{s t_{r e l}}{s t_{r e l}+a}\right)^{b}$ 


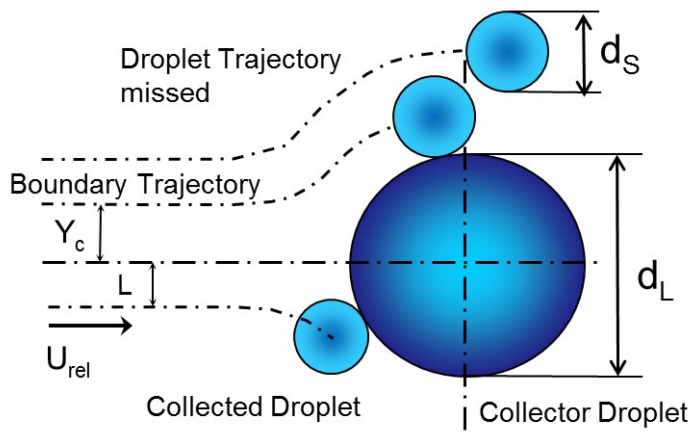

Figure 5: Illustration of inertial impact efficiency for the collision of a small droplet with a larger collector droplet.

In the work of Schuch and Löffler (1978) it was shown that the impact efficiency for inertial impaction is depending on the large droplet Reynolds-number determined with the relative velocity. This is caused by the fact that with increasing collector Reynolds number the flow deflection is increasingly compresses towards the collector. The resulting different values for the constants $a$ and $b$ are provided in Table 1 . The results of the different correlations are given in Figure 6 together with own simulations which agree fairly well. For a given relative Stokes-number the increase in Reynolds-number yields growing impact efficiency (Sommerfeld and Lain 2009) due the raising small particle inertia with respect to the stronger flow deflection. Eventually, a collision is only occurring if the randomly drawn lateral displacement $L$ (see Sommerfeld 2001) is smaller than the value of $\left(Y_{c}+d_{L}\right)$. Note that the lateral displacement $L$ is identical to the impact parameter $B$.

\begin{tabular}{|c|c|c|}
\hline $\mathrm{Re}_{\mathrm{c}}$ & $\mathrm{a}$ & $\mathrm{b}$ \\
\hline$>1$ & 0.25 & 2.0 \\
\hline $60-80$ & 0.506 & 1.84 \\
\hline 40 & 1.03 & 2.07 \\
\hline $10-20$ & 1.24 & 1.95 \\
\hline$<1$ & 0.65 & 3.7 \\
\hline
\end{tabular}

Table 1: Constants for the correlation proposed by Schuch and Löffler (1978) for the impact efficiency (Eq. (8).

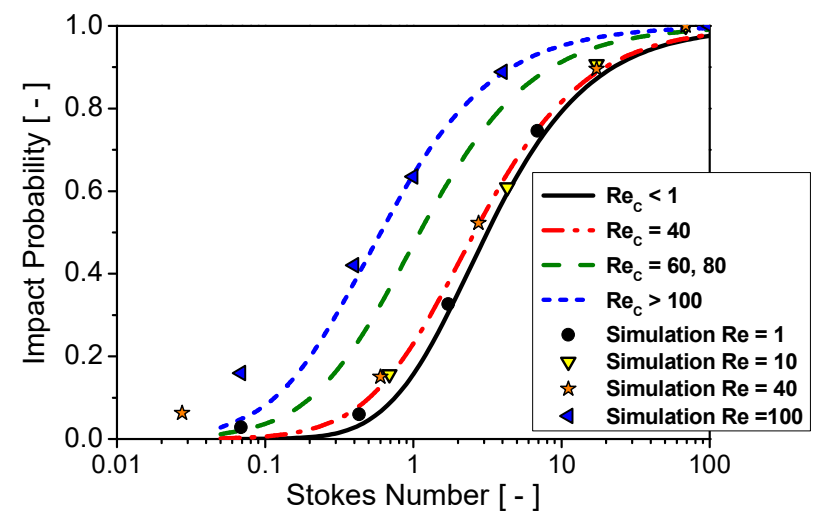

Figure 6: Impact efficiency as a function of relative Stokes number, lines: correlations proposed by Schuch and Löffler (1978); symbols: present numerical calculations.

The above model for considering the impact efficiency may be only applied to turbulent flows if the collision complex is much smaller than the smallest turbulent eddies, namely the Kolmogorov length scale. Otherwise of course the impact efficiency is still very important, but the collision geometry is much more complex. In such a situation the trajectories of the small colliding droplets are affected by turbulent transport, so that even small droplets moving initially outside the collision cross-section might hit the collector. Thereby the impact efficiency will rise again when reducing the Stokes number. This will also happen if the colliding droplets are very small so that their motion is affected by Brownian dispersion. 
The final step in the droplet collision model is the determination of the collision outcome, based on the collision maps $B=f(\mathrm{We})$ as it was described above (Figure 1$)$ and the theoretically derived boundary lines. As indicated in Figure 1 three characteristic points are identified to allow a generalisation of the collision map with regard to droplet fluid properties, mainly viscosity and size ratio $\Delta$.

\section{Characteristic points in the collision maps}

From the collision map in Figure 1 it is obvious that there are three characteristic points which could be used for describing the change of the collision maps for example with droplet size ratio or increasing fluid viscosity. The first point is the We-number at $B=0$ for the transition from coalescence to reflexive separation. The second is the triple point where bouncing, stretching separation and coalescence meet. This point was identified by Sommerfeld and Kuschel (2016) and Sommerfeld (2016) to have a characteristic movement with increasing viscosity. The third characteristic point results from the lower boundary of bouncing intercepting with the horizontal axis (Estrade et al. 1999). In some studies and for some liquids also a coalescence region for very low We over a wide range of impact parameters was found (Qian and Law 1997, Chen 2007 and Huang and Pan 2015), which however will not considered here for the moment.

For collisions of identical sized droplets the critical Weber-number $W e_{C}$ has been quite often identified to shift to the right with increasing Ohnesorge-number and Qian and Law (1997) found a good correlation with minor scatter considering also various literature data (e.g. Jiang et al. 1991):

$W e_{C}=\sqrt{2} 480 \mathrm{Oh}+15$

Also the experiments of Huang and Pan (2015) for different alkanes followed this correlation for both 300 and 600 $\mu \mathrm{m}$ droplets. Actually, there is no difference for the location of the critical Weber-number when plotting the data versus the droplet Ohnesorge-number. Very high viscous droplets up to $58 \mathrm{mPa} \cdot \mathrm{s}$ were considered by Gotaas et al. (2007), namely monoethylenglycol (MEG), diethylenglycol (DEG) and triethylenglycol (TEG), yielding Ohnesorge numbers up to 0.34 . For the low Oh-regime they proposed a similar correlation as above:

$W e_{C}=643 \mathrm{Oh}+14.8$

Moreover, they suggested an additional correlation for Ohnesorge-numbers larger than about 0.045 using also experimental data from Willis and Orme (2003):

$W e_{C}=9309 \mathrm{Oh}^{1.706}$

In the droplet collision studies of Kuschel and Sommerfeld (2013) several solutions were used as liquid with viscosities between 1 and $50 \mathrm{mPa} \cdot \mathrm{s}$, yielding up to $\mathrm{Oh}=0.37$. However, since here the maximum We-number was about 100, not for all cases the critical Weber-number could be identified. Hence the non-linear correlation proposed by Gotaas et al. (2007) fits the high-viscosity data reasonably well (Figure 7 a) including silicon oils, MEG and DEG and a VOF (volume of fluid) simulation of Xia and Hu (2015) for $10 \mu \mathrm{m}$ liquid aluminium droplets $(\mathrm{Oh}=0.115)$. The critical Weber-number for mixtures of Diesel/Biodiesel and Diesel/water emulsions (Chen et al. 2016) are however remarkably over-predicted by this correlation, similar to solution droplets of Kuschel and Sommerfeld (2013).

Now we turn to the low Oh-regime wherefore numerous data are available (Figure $7 \mathrm{~b}$ ). Here only the initial correlation proposed by Qian and Law (1997) is shown for comparison. Most of the data for the critical Webernumber are lying around and along this correlation. However some are a bit more off the line, but it is not possible to specify the reason for that at the moment. Here further experiments are needed for clarifying this deviation. Two data point for the reference oil FVA1 at low temperature (Sommerfeld and Kuschel 2016) give almost double the value as resulting from the correlation. Similarly higher viscous alcohols are lightly above the line (Sommerfeld and Kuschel 2016). Interestingly, all VOF simulations for water droplets (Saroka et al. 2012) of different size are clustered above the correlation, which indicates that some phenomena are not captured properly by these simulations. One result of Rabe et al. (2010) for different sized water droplets is also found in this region. This point is just included for illustrating the fact that decreasing size ratio yields a shift of the triple point as well as $W e_{c}$ to the right (see Tang et al. 2012). The data points for the Diesel/water emulsion droplets are initially above and for higher water content, and hence larger viscosity, below the correlation, which suggests a slightly lower slope of such correlation (Chen et al. 2017). Consequently, the correlation proposed by Qian and Law (1997), Eq. 9 , is still a very good approximation to describe the shift of the critical We-number with changing fluid properties up to $\mathrm{Oh} \approx 0.1$. It seems that higher viscous liquids may be described better by the Gotaas et al. (2007)correlation.

It was shown previously that the adapted correlation of Ashgriz and Poo (1990) predicts the lateral extent of reflexive separation (i.e. the boundary line separating reflexive separation and coalescence) very well for many 
cases (Sommerfeld and Kuschel 2016). Hence the original correlation (Eq. 5) is just shifted by the critical Wenumber:

$W e=\left(W e_{C}-W e_{C, w}\right)+3\left[7\left(1+\Delta^{3}\right)^{2 / 3}-4\left(1+\Delta^{2}\right)\right] \frac{\Delta\left(1+\Delta^{3}\right)^{2}}{\Delta^{6} \eta_{S}+\eta_{L}}$

It is known from various studies summarised above, that the critical Weber-number also is shifted to higher Weber-numbers when the size ratio $d_{S} / d_{L}$ is decreasing. Theoretical correlations for $W_{C}$ in dependence of size ratio also considering different liquids were provided by Tang et al. (2012).
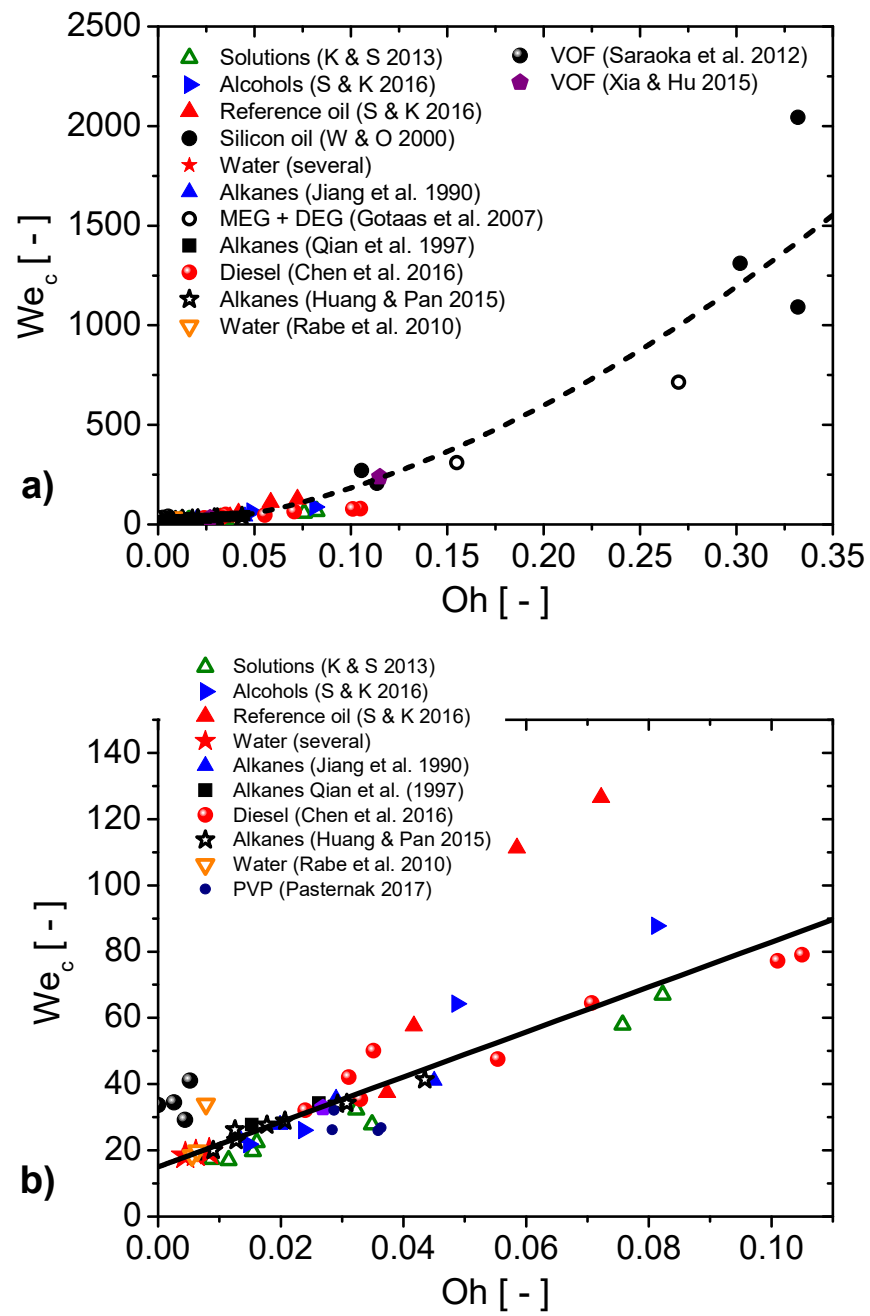

Figure 7: Summary of the critical Weber-number for the full (a) and small (b) regime of Ohnesorge-numbers obtained from different experimental studies using a range of different liquids (K \& S 2013: Kuschel and Sommerfeld (2013); S \& K 2016: Sommerfeld and Kuschel (2016); W \& O 2000: Willis and Orme (2000)), dashed line correlation Eq. (11), closed line correlation Eq. (9).

The next characteristic point is the triple point location determined by the Weber-number $W e_{T}$. In the study of Sommerfeld (2016) the own measurements regarding the location of the triple point showed with growing viscosity and hence Ohnesorge-number first an increase of $\mathrm{We}_{\mathrm{t}}$ followed by a decrease beyond about $\mathrm{Oh}=0.1$. A suitable correlation between $W e_{T}$ and $\mathrm{Oh}$ could be elaborated based on a very good coherence of the data in a $\mathrm{Ca}=\mathrm{f}(\mathrm{Oh})$ diagram.

$\mathrm{Ca}=-0.0088+5.4246 \mathrm{Oh}-2.2036 \mathrm{Oh}^{2}$

The available experimental results together with the proposed correlation regarding the triple point location are summarized in Figure 8 for the lower Oh-regime. Here the capillary number is obtained from the available data on We and Oh through $\mathrm{Ca}=\mathrm{Oh} \sqrt{W e}$. Most of the data are nicely scattered around the correlation line except one for Diesel with $60 \%$ of Biodiesel (blue star at Oh $=0.11$ ). 


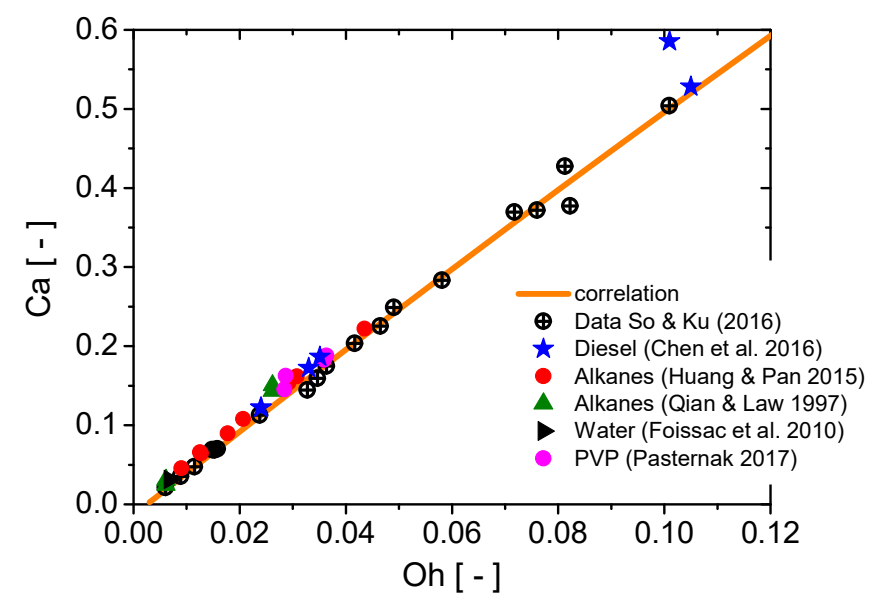

Figure 8: Triple point location from different experimental data (So \& Ku (2016) includes all data presented by Sommerfeld and Kuschel 2016) shown as $\mathrm{Ca}=\mathrm{f}(\mathrm{Oh})$ with best fit correlation given in Eq. (13).

Having now a correlation for the triple point Weber-number in dependence of the Oh-number, which only depends on the material properties of the droplets, it is possible to adapt a boundary line model (in this case the Jiang et al. (1992) model) in such a way that it goes though the triple point ( $B_{T}$ and $W e_{T}$ need to be known from experiments) and thereby matches the experimentally observed boundary between coalescence and stretching separation. If a bouncing region is observed left of the stretching separation region, which was reported by many measurements for alcohols, oils, alkanes and solutions, the triple point is also the starting point of the boundary line. An exception is water where this line extends to $B=1$ at $W e=0$ separating bouncing and coalescence (see e.g. Kuschel and Sommerfeld 2013). This matching procedure was successfully achieved with the Jiang et al. (1997) model which accounts for the effect of viscous dissipation (Sommerfeld 2016). By setting the model constant $C_{b}=1$ (this value was obtained for higher relaxation velocities $u_{\text {relax }}=\sigma / \mu$ by Sommerfeld and Kuschel 2016) it was found that the model parameter $\mathrm{C}_{a}$ could be very well correlated with the Ohnesorge number for pure fluids and solution droplets. For pure fluids the following correlation was found:

$\mathrm{C}_{\mathrm{a}}=2.3-11.12 \cdot \mathrm{Oh}+23.74 \cdot \mathrm{Oh}^{2}-18.2 \cdot \mathrm{Oh}^{3}$

A validation of this modelling approach was presented for higher viscous liquids by Sommerfeld (2017) and Diesel as well as solution droplets by Sommerfeld (2016).

\section{Spray calculations}

For analysing the effect of impact efficiency and the occurring droplet collision outcomes the experimentally studied single spray of Rüger et al. (2000) was considered using first water as liquid. In the measurements a hollow cone spray nozzle with a spread angle of 45 degree was centrally mounted into the upper part of a 400 $\mathrm{mm}$ diameter pipe. The test section had a length of about 1 meter (Figure 9). For avoiding any recirculation region within the test section, a low airflow was forced through the pipe by a suction blower. Above the spray nozzle a honeycomb was installed in order to homogenise the flow. The nozzle was operated at a water flow rate of 0.135 $\mathrm{I} / \mathrm{min}$. Air and droplet velocity profiles at the inlet plane, which was located $25 \mathrm{~mm}$ downstream of the nozzle exit, were measured by a two-component phase-Doppler anemometer. Here droplets with a size less than $2.5 \mu \mathrm{m}$ were identified as tracer particles for the air phase velocities over the entire inlet cross-section. Of course droplets were only present just underneath the spray nozzle exit. Here local droplet size distributions were measured from the centre location of $z=0 \mathrm{~mm}$ up to a radial location of $z=30 \mathrm{~mm}$ at intervals of $1 \mathrm{~mm}$. Therefore, as the inlet condition for the droplet phase besides the droplet size distribution also size-velocity correlations were available for the axial and radial components. The mean tangential velocity component was close to zero. Hence, for each injected parcel a size was drawn from the local cumulative distribution function by a random process. The sizevelocity correlations provided the droplet mean velocities. From the correlation between the size and rms-values the standard deviations for each velocity component was obtained. The instantaneous droplet fluctuating velocities were then randomly drawn from the corresponding normal distribution functions. 

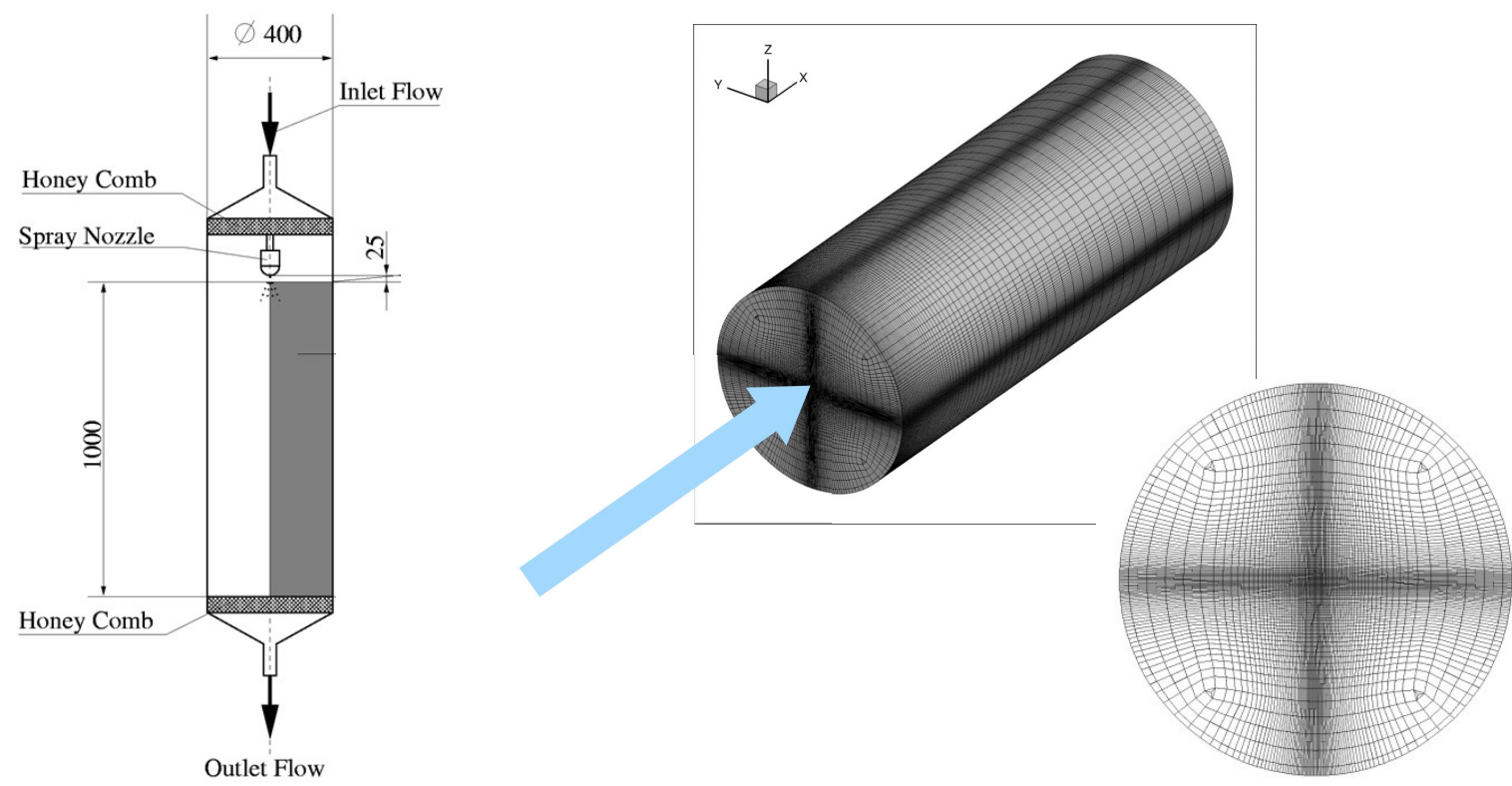

Figure 9: Illustration of the spray facility with major dimensions and spray nozzle location (left) and sketch of the numerical grid used for the computations with about 700,000 control volumes (middle) as well as a cross-sectional view of the grid (right).

The numerical grid used for the fully three-dimensional calculations is also shown in Figure 9 (middle) with the direction of spray injection. The employed grid was a structured O-grid divided in 5 blocks with in total 700,000 control volumes covering the region from the inlet plane $25 \mathrm{~mm}$ downstream the nozzle $(x=0 \mathrm{~mm})$ up to the outlet $1025 \mathrm{~mm}$ downstream the nozzle exit $(x=1000 \mathrm{~mm})$. In order to resolve the spray nozzle injection region sufficiently the grid was remarkably refined (i.e. indicated by the cross-like pattern in Figure 9 right). The numerical computations were done for a steady-state mode where after reaching a converged fluid flow solution 372,000 parcels were sequentially injected at the inlet and tracked through the flow field. Thereafter, the obtained source terms were coupled back to the fluid flow calculations with under-relaxation which then was recalculated until convergence was reached. Eventually about 35 coupling iterations were required to obtain a coupled converged result of the spray.

Since the calculations were first conducted for water according to the measurements, the three boundary line model was used for separating the collision outcome regimes. The boundary line reflexive separation/coalescence was used according to Ashgriz and Poo (1990) given in Eq. (5). As it best fits for water, also the stretching separation/coalescence boundary was that of Ashgriz and Poo (1990) provided in Eq. (3). Finally the lower boundary of bouncing uses the Estrade et al. (1999) correlation (Eq. 1) with the shape factor of $\Psi=3.351$. This boundary line ended of course at the intersection with the stretching/coalescence boundary. Although in the spray simulations collisions occur with a wide range of size ratios, all correlations were used with a fixed size ration of $\Delta=1.0$ for clarity.

As a first step the simulations were validated against the experiments and the agreement of all gas and droplet phase properties was found quite well similar to the comparison published by Rüger et al. (2000). The numerical calculations with droplet collisions were conducted by storing all the collision events occurring. In Figure 10 the total collision events are summarized and also the numbers for bouncing, coalescence and stretching as well as reflexive separation are given. The total collision number is 2.45 million when assuming $100 \%$ impact efficiency. Considering reduced impact efficiency through the motion of small droplet around larger ones upon interaction, only 1.66 million collisions are counted. This demonstrates that the collision rate is over-predicted by $48 \%$ when not considering impact efficiency, which is of course a huge error.

Looking at the number of collisions with respect to collision type, it is clear that coalescence is dominating with about $88 \%$ of the total collisions in both cases, i.e. without and with impact efficiency (Figure 10). Coalescence events occur for a relatively wide region near the nozzle exit, but continue to happen up to the end of the computational domain (Figure $11 \mathrm{~b}$ ) but with reduced probability. The reduction of the collision probability along the spray is of course associated with a decrease of relative velocity and local droplet concentration. Droplet size distributions only slightly change along the spray although coalescence is happening. The second probable collision event is bouncing (Figure 10) occurring mainly in the initial region of the spray (Figure 11). Stretching separation happens of course only if the We-number is exceeding the triple point value, hence can only take 
place close to the nozzle where the relative velocity is still high (Figure 11). The probability of reflexive separation is even lower and also occurs only near the nozzle exit.

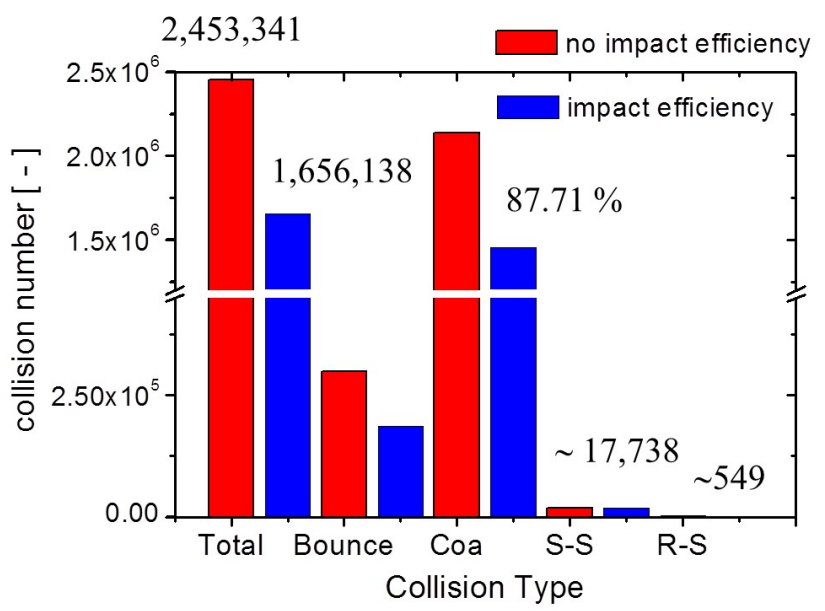

Figure 10: Droplet collision counts throughout the entire spray, comparing a calculation without and with impact efficiency and showing the numbers of collisions resulting in bouncing, coalescence and stretching as well as reflexive separation.

a)

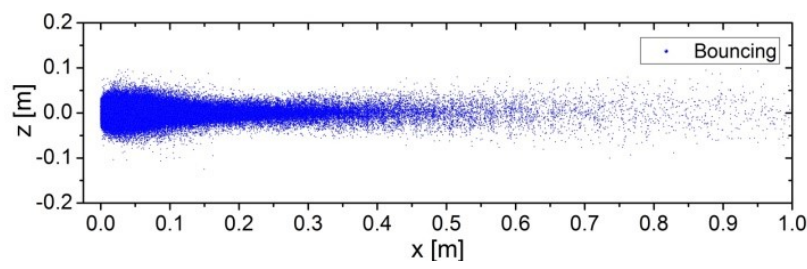

b)

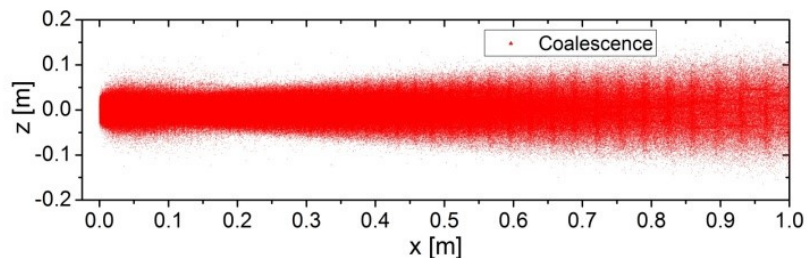

c)

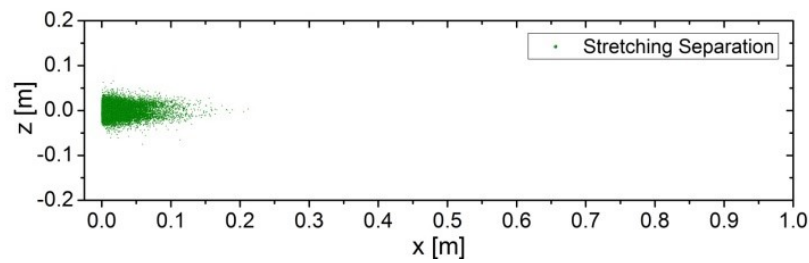

Figure 11: Scatter plot of the location of collision events throughout the spray, a) locations of bouncing, b) locations of coalescence, c) locations of stretching separation.

From the simulations with impact efficiency also the droplet size ratio upon collision was recorded along the spray for different cross-sections. This result shows that preferentially small and large droplets collide due to the higher relative velocity in a spray (Figure 12). Hence, the maximum in the PDF of the size ratio has initially a peak around 0.18 which is further downstream spread to values between 0.2 and 0.3 . It should be noted that such small size ratios have never been analysed experimentally for confirming the above introduced boundary lines.

\section{Conclusions}

An attempt was made for generalising the collision maps describing the outcome of binary collisions between droplets with respect to liquid properties. The triple point location was obtained through a correlation between $\mathrm{Ca}$ $=\mathrm{f}(\mathrm{Oh})$ based on numerous available experiments. As a boundary line to capture viscosity effects, the Jiang et al. (1992) correlation was used. The involved parameters could be adapted properly through a dependence on the Ohnesorge number for pure liquids and solutions. The upper boundary of reflexive separation was demarked through the Ashgriz and Poo (1990) correlation. With increasing viscosity the critical We-number is shifted to the right which could be also obtained with a correlation on the Ohnesorge-number. The lower boundary of bouncing 
Estrade et al. 1999) could not yet be properly adapted with respect to viscosity effects. The three-line boundary model was applied in Euler/Lagrange calculations of a turbulent water spray. It was shown that neglecting the impact efficiency yields an over-prediction of the collision rate by about $48 \%$. The analysis of the collision events showed that coalescence and then bouncing were the most frequent outcome. Moreover it was shown that the most frequent size ratio upon collision has a value of about $\Delta \approx 0.2$. Experimental studies with such a size ratio have not yet been performed. Therefore, it is believed that the discussed boundary lines cannot cover such small size ratios.

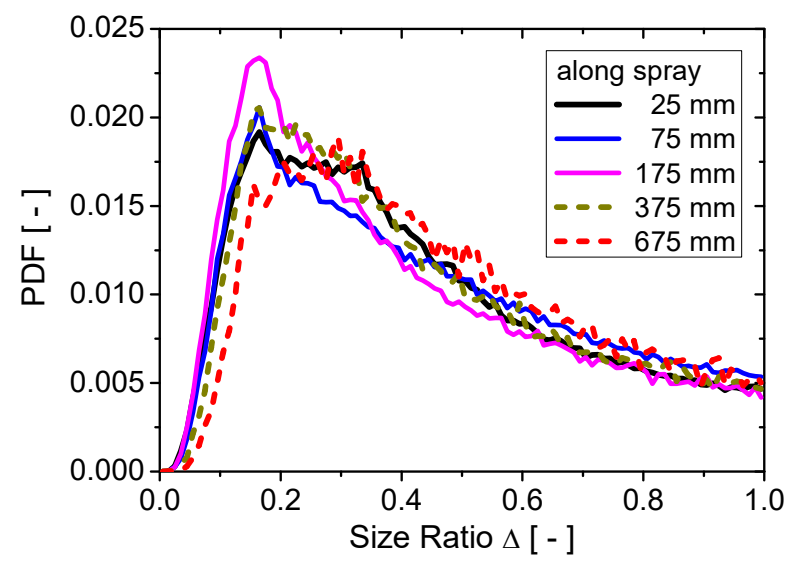

Figure 12: Cross-sectional averages of the PDF (probability density function) for the droplet size ratio upon occurrence of a collision along the simulated spray.

\section{Acknowledgements}

The authors acknowledge the financial support of this research project by the Deutsche Forschungsgemeinschaft (DFG) under contract SO 204/35-1 to 3.

\section{References}

Ashgriz, N.; Poo, J.Y.: Coalescence and separation in binary collisions of liquid drops. Journal of Fluid Mechanics, Vol. 221, $183-204$ (1990)

Brazier-Smith, P. R., Jennings, S. G. and Latham, J.: The interaction of falling water drops: coalescence. Proc. R. Soc. Lond. A, Vol. 326, 393 - 408 (1972)

Chen, R.-H.: Diesel-diesel and diesel-ethanol drop collisions. Applied Thermal Engineering, Vol. 27, 604 - 610 (2007)

Chen, R.-H., Wang, W.-C. and Chen, Y.-W.: Like-drop collisions of biodiesel and emulsion diesel. European Journal of Mechanics B/Fluids, Vol. 60, 62 - 69 (2016)

Crowe, C.T., Schwarzkopf, J.D., Sommerfeld, M. and Tsuji, Y.: Multiphase Flows with Droplets and Particles. $2^{\text {nd }}$ Edition, CRC Press, Boca Raton, U.S.A. (2012), ISBN 978-1-4398-4050-4.

Estrade, J.-P., Carentz, H., Lavergne, G., Biscos, Y.: Experimental investigation of dynamic binary collision of ethanol droplets - a model for droplet coalescence and bouncing. International Journal of Heat and Fluid Flow, Vol. 20, 486 - 491 (1999)

Gavaises, T.L. Theodorakakos, A. Bergerles, G. and Brenn, G.: Evaluation of the effect of droplet collisions on spray mixing. Proc. Inst. Mechanical Engineers, Vol. 210, 465 - 465 (1996)

Gotaas, C., Havelka, P. Jakobson, H. A. and Svendsen H.F, Hase, M., Roth, N., and Weigand, B.: Effect of viscosity on droplet-droplet collision outcome Experimental study and numerical comparison. Physics of Fluids, Vol. 19, 102106 (2007 a)

Guo, B. Fletcher, D.F. and Langrish, T.A.G.: Simulation of the agglomeration in a spray using Lagrangian particle tracking. Applied Mathematical Modelling, Vol. 28, 273 - 290 (2004)

Ho, C.A. and Sommerfeld, M. (2002) Modelling of micro-particle agglomeration in turbulent flow. Chem. Eng. Sci., Vol. 57, $3073-3084$.

Huang, K.L. and Pan, K.L.: Size effect on the transition from coalescence to bouncing regime in binary droplet collision. ICLASS 2015, $13^{\text {th }}$ Triennial International Conference on Liquid Atomization and Spray Systems, Tainan, Taiwan, August 23-27, 2015

Jiang, Y. J., Umemura, A. and Law, C.K.: An experimental investigation on the collision behavior of hydrocarbon droplets. Journal of Fluid Mechanics, Vol. 234, 171 - 190 (1992) 
Kohnen, G., Rüger, M. and Sommerfeld, M.: Convergence behaviour for numerical calculations by the Euler/Lagrange method for strongly coupled phases. In: "Numerical Methods in Multiphase Flows 1994, (Eds. C.T. Crowe et al.), ASME, New York, FED-Vol. 185, 191-202 (1994)

Kuschel, M. and Sommerfeld, M.: Investigation of droplet collisions for solutions with different solids content. Experiments in Fluids, Vol. 54, 1440 (2013)

Laín, S. and Sommerfeld, M.: Euler/Lagrange computations of pneumatic conveying in a horizontal channel with different wall roughness, Powder Technology, Vol. 184, 76-88 (2008)

Lain, S. and Sommerfeld, M.: Numerical calculation of pneumatic conveying in horizontal channels and pipes: Detailed analysis of conveying behaviour. International Journal of Multiphase Flow, Vol. 39, 105-120 (2012)

Munnannur, A. and Reitz, R. D.: A new predictive model for fragmenting and non-fragmenting binary droplet collisions. Int. J. Multiphase Flow, Vol. 33, 873 - 896 (2007)

Nijdam, J.J., Guo, B., Fletcher, D.F. and Langrish, T.A.G.: Lagrangian and Eulerian models for simulating turbulent dispersion and coalescence of droplets within a spray. Applied Mathematical Modelling, Vol. 30, 1196 1211 (2006)

O’Rourke P.J.: Collective drop effects on vaporizing liquid sprays. Dissertation, Los Alamos National Laboratory, New Mexico (1981)

Post, S. L. and Abraham, J.: Modeling the outcome of drop-drop collisions in Diesel sprays. International Journal of Multiphase Flow, Vol. 28, 997 - 1019 (2002)

Qian, J. and Law, C.K.: Regimes of coalescence and separation in droplet collision. Journal of Fluid Mechanics, Vol. 331, 59 - 80 (1997)

Rabe, C., Malet, J. and Feuillebois: Experimental investigation of water droplet binary collisions and description of outcomes with a symmetric Weber number. Physics of Fluids, Vol. 22, 047101 (2010)

Rüger, M., Hohmann, S., Sommerfeld, M. and Kohnen, G. Euler/Lagrange calculations of turbulent sprays: The effect of droplet collisions and coalescence. Atomization and Sprays, Vol. 10, $47-81$ (2000)

Saroka, M.D. Ashgriz, N. and Movassat, M: Numerical Investigation of Head-on Binary Drop Collisions in a Dynamically Inert Environment. Journal of Applied Fluid Mechanics, Vol. 5, 23-37 (2012)

Schuch, G., Löffler, F.: Über die Abscheidewahrscheinlichkeit von Feststoffpartikeln an Tropfen in einer Gasströmung durch Trägheitseffekte. Verfahrenstechnik Vol. 12, 302-306 (1978)

Schiller, L., and Naumann, A. (1933) Uber die grundegenden Berchnungen bei der Schwerkraftaufbereitung, $Z$. Ver. Deut. Ing., 77, 318-320.

Sommerfeld, M.: Modellierung und numerische Berechnung von partikelbeladenen turbulenten Strömungen mit Hilfe des Euler/Lagrange-Verfahrens. Habilitationsschrift, Universität Erlangen-Nürnberg, Shaker Verlag, Aachen (1996)

Sommerfeld, M. (2001) Validation of a stochastic Lagrangian modelling approach for inter-particle collisions in homogeneous isotropic turbulence". Int. J. of Multiphase Flows, Vol. 27, 1828-1858.

Sommerfeld, M., van Wachem, B. \& Oliemans, R. (2008 a) Best Practice Guidelines for Computational Fluid Dynamics of Dispersed Multiphase Flows. ERCOFTAC, ISBN 978-91-633-3564-8.

Sommerfeld, M. and Lain, S.: From elementary processes to the numerical prediction of industrial particle-laden flows. Multiphase Science and Technology, Vol. 21, 123 - 140 (2009)

Sommerfeld, M. and Kuschel, M.: Modelling droplet collision outcomes for different substances and viscosities. Experiments in Fluids, Vol. 57, 187 (2016)

Sommerfeld, M.: Modelling separation processes of colliding droplets. ILASS - Europe 2016, 27 $7^{\text {th }}$ Annual Conference on Liquid Atomization and Spray Systems, 5. - 7. Sep. 2016, Brighton, UK

Sommerfeld, M. (2017) Lagrangian Modelling of Droplet Collisions in Spraying Systems. Proceedings of the European Combustion Meeting, Dubrovnik, 18. - 21. April 2017

Sundaram, S., and Collins, L. R.: Numerical Considerations in Simulating a Turbulent Suspension of FiniteVolume Particles. J. Comp. Phys. Vol. 124, 337-350 (1996).

Tang, C.G., Zhang, P. and Law, C.K.: Bouncing, coalescence, and separation in head-on collision of unequal-size droplets. Physics of Fluids, Vol. 24, Paper 022101 (2012)

Willis, K. and Orme, M.: Binary droplet collisions in a vacuum environment: an experimental investigation of the role of viscosity. Experiments in Fluids, Vol. 34, 28 - 41 (2003)

Xia, S.-Y. and Hu, C.-B.: Numerical investigation of head-on binary collision of alumina droplets. Journal of Propulsion and Power, Vol. 31, 416-428 (2015) 